\title{
Genital Hygiene and Strategies for HPV Prevention
}

\author{
Jeevitaa Kshersagar and Meghnad G Joshi* \\ Department of Stem Cells \& Regenerative Medicine, DY Patil University, India
}

*Corresponding author: Meghnad G Joshi, Associate Professor and Head of the Department, Department of Stem Cells and Regenerative Medicine, DY Patil University, D Y Patil Vidyanagar, Kasaba Bawda, Kolhapur -416006, Maharashtra, India

Submission: 非 February 13, 2018; Published: 非 February 26, 2018

Keywords: Cervical cancer, Genital hygine, HPV infection, HPV vaccine

\section{Opinion}

Cervical cancer (CC) is $80 \%$ of global burden, the second most common cancer in women worldwide and leading cancer in Indian women. Approximately 40 Human papillomavirus (HPV) types infect cervix through sexual transmission [1,2]. HPV is a chronic disease and product of infection of sexually active women along with poverty, lower education level, low standards, multiparity, multiple sexual partners, using oral contraceptive pills, tobacco smoking, illiteracy, malnutrition and poor genital hygiene, dietary deficiencies of vitamins, co-infection with HIV, Herpes simplex virus type 2, Chlamydia trachomatis, bacterial vaginosis immunosuppressant drugs are all co-factors that progress from HPV infection to CC [3]. These co-factors are un-addressed widespread issues in the undeveloped sectors of the world. Intervention to increase program to prevent the development of unhealthy life behaviors and reduce the non-HPV risk factors can have immense impact on decreasing morbidity and mortality of genital malignancies and many preventable communicable and non-communicable human ailments [4]. It is unrecognized infection without any specific discern signs and symptoms. The persistent infections are phylogenetically related to either HPV 16 (serotype $31,33,35,52$, and 58) or HPV 18 (serotype 39, 45, 59, and 68) [5]. It is the most important risk factor for cervical intraepithelial neoplasia and invasive cervical cancer. HPV serotypes 16 and 18 account for nearly $76.7 \%$ of CC in India. CC occurs early and strikes at the productive period of women with rise in incidence in 3034 years of age and peaks at 55-65 years, with a median age of 38 years. More than $80 \%$ of the sexually active women acquire HPV infection by 50 years of age.

Attention must be directed towards other factors, which have aforementioned risk factors of CC. Till date, enough research has not been done to determine the direct link between HPV and personal genital hygiene. Genital hygiene play a role in the decreasing the chances of yeast infections, bacterial vaginosis and HPV [6]. There is a lack of consensus of HPV infection with respect to reproductive and genital health. Shaw E et al. [7] examined the genital health determinants related to HPV infection by Cohort study. Use of contraceptive injections and oral contraceptive pills was associated with increased risk of HPV infection. Use of tampon, previous gynecologic infections, pelvic inflammatory diseases and cervical inflammation were associated with increased risk of HPV infection. This editorial focuses on the co-relation of contraceptives and genital health with cervical HPV infections and to focus on importance of HPV vaccine [7].

The continuous use of single tampons and sanitary pads may be responsible for transient and persistent HPV infections. The absorbent pads contain fibers, cellulose gels, Bisphenol A. (BPA) and Bisphenol S. (BPS) plastic chemicals that can lead to harmful complications. Pthalates, an ester of phthalic acid used to increase the flexibility of BPA and BPS is responsible for disregulation of genes. These pads are not purely cotton made. The synthetic fibers and rayon used as absorbent. The ultra white appearance of pads is due to chlorine bleaching used for cotton. When bleached, rayon releases dioxin, which can cause pelvic inflammatory disease, if not treated properly, may lead to CC. There is one more possibility that Furan, a dangerous pesticide chemical sprayed to harvest the cotton can definitely cause harm as Furan is linked to thyroid malfunction, cancer, infertility and many other health problems in experimental animals. Further Prolonged use of sanitary pad encourage bacterial growth, is responsible for an overgrowth of staphylococcus aureus bacteria which releases toxins and results into severe allergies and yeast infections. Gynecologic concurrent infections were also associated with a significant increase in prevalence of HPV infections. Further studies are necessary to confirm the low to moderate associations observed with respect to genital infections due to pads [8-10].

Condoms are effective in preventing HIV and STD infections among both men and women. Rachel $\mathrm{L}$ et al. demonstrated an inverse, temporal association between the frequency of condom use 
by male partners and the risk of HPV infection in women suggesting a causal, protective effect. In particular, several studies have found that condom use by men does not reduce the risk of HPV infection in women and the incidence of other sexually transmitted infections are more limited as condom use has tropism for vagina and may increase the risk of Subgenus $3 \mathrm{HPV}$ infections. It is impossible to accurately assess the role of condoms in preventing infections. The American College of Obstetricians and Gynecologists suggested that there is a need for studies to determine the degree to which condoms prevent acquisition of HPV infection or development of HPV-related sequelae [11-13].

The injectable hormonal contraceptive depomedroxyprogesterone acetate (DMPA) has been associated with increased risk of viral infection acquisition and other infections, but findings are inconsistent. Whether DMPA increases the risk of other sexually transmitted viral infections is unknown. Bacterial vaginosis has been positively associated with HPV infections as lack of protective lactobacilli can increase susceptibility of Chlamydia trachomatis, Neisseria gonorrhoeae, HIV and HPV infections. Women with previous HPV-related infection have an increased risk of re-infection due to sexual behaviors. There may be a possible relation between chronic inflammation and cervical carcinogenesis and may play a role in increasing risk of HPV infection [14].

\section{Concerns and Safety of Cervical Cancer}

Every year more than 250,000 people die from HPV infection. The Food and Drug Administration (FDA) approved a vaccine that protects against certain strains of HPV infection and it is emerging as an effective option. Hence, importance of HPV vaccine has stirred much excitement as well as debate. CC cytology screening substantially reduced cervical cancer incidence. In uninfected cases, anti-cancer vaccine provides specific protection against HPV 16 and 18. In recent years, periodic cervical exfoliated cytologic evaluation and detection of HPV DNA or RNA in cervico-vaginal cytological or tissue specimen can detect the CC. Exfoliative cytology is well accepted by patients as it is less invasive and simple, rapid technique and is suitable for routine population screening programs. In the recent past, Raman spectroscopy (RS) of exfoliated cervical cells has been explored for diagnosis. In vivo RS in oral and cervix cancers has previously proved promise in management of both. Hole A et al., explored the cervix specimens by Raman microprobe and found exfoliated cells may be effective patient-friendly, non-invasive, rapid method for management of cervix cancers. To reduce the number of CC patients, there is immense need to increase the genital hygiene educational standards and health status of the populations $[15,16]$.

Routine PAP screening is used to find cellular abnormalities in cervical tissue, aiding early diagnosis. An abnormal Papanicolou (Pap) smear is $35 \%$, CIN $20 \%$ and ICC is $<1 \%$ approximately however, in women without routine screening, is up to $4 \%$. HPV is a necessary but not a sufficient cause. Other than above mentioned co-causes, viral load and viral integration are likely to be important, but have not been clearly identified. From above literature, there is no clear evidence that barrier methods of contraception confer a protection against HPV infection. HPV infections can be prevented except by abstinence and lifetime mutual monogamy. The HPV vaccination is of public health importance, safe and efficacious. Routine three doses of HPV vaccine recommend at early age prior to sexual debut as response to the vaccine is better at younger ages than it is at older ages. In older age, the women may carry HPV infection during her active sexual life. So that vaccines are not $100 \%$ protective against CC. but it may protect from the further infections. Hence, screening programs should continue. The quadrivalent vaccine, Gardasil has demonstrable efficacy against vaginal and vulvar cancers and protects against anogenital warts also. None of the vaccines can treat an existing HPV infection but protect from specific strains of HPV haven't been exposed to already.

The primary obstacle to vaccination is financial because of the high cost. The affordability and accessibility of these vaccines is a major concern for a mass vaccination program in developing countries like India although results in the development of vaccines against HPV are promising. Duration of protection, need for boosters, effect on prevalence and incidence of HPV types in different population, safety and immunogenicity of simultaneous administration with other vaccines has to be looked into. The role of routine HPV vaccine in males has to be studied in detail. HPV vaccination and regular screening, prompt medical attention is the most effective to prevent CC. The girls should be educated the importance of genital hygiene and vaccination in their school life so that it will help to aware the population at proper age and will definitely reduce the incidence of persistent infection with these HPV types.

This study was supported by a grant to Dr. Meghnad G Joshi from Department of Science and Technology (DST), Govt. of India (SB/SO/HS/0198/2013).

\section{References}

1. Sundar S, Singh PK, Frampton J, Trimble E, Rajaraman P, et al. (2018) Harnessing genomics to improve outcomes for women with cancer in India: key priorities for research. Lancet oncology 19(2): e102-e112.

2. Giuliano AR, Nyitray AG, Kreimer AR, Pierce Campbell CM, Goodman MT, et al. (2015) EUROGIN 2014 roadmap: Differences in human papillomavirus infection natural history, transmission and human papillomavirus-related cancer incidence by gender and anatomic site of infection. Int J Cancer 136(2): 2752-2760.

3. Nayak UA, Murthy SN, Swarup A, Dutt V, Muthukumar V (2016) Current knowledge, attitude, and practice about cervical cancer among rural Indian women. International Journal of Medical Science and Public Health 5(8): 1554-1558.

4. Shield KD, Parkin DM, Whiteman DC, Rehm J, Viallon V, et al. (2016) Population Attributable and Preventable Fractions: Cancer Risk Factor Surveillance, and Cancer Policy Projection. Curr Epidemiol Rep 3(3): 201-211.

5. Hesselinka AT, Berkhofb J, Salma ML, Spluntera AP, Geelenc TH, et al. (2014) Clinical Validation of the HPV-Risk Assay, a Novel Real-Time PCR Assay for Detection of High-Risk Human Papillomavirus DNA by Targeting the E7 Region. J Clin Microbiol 52(3): 890-896.

6. Chouhan S, Malik R, Nigam R, Jain P (2016) Immunohistochemical and polymerase chain reaction study on expression of hpv and hsv and its histopathological pattern correlation in cervical lesions. J Evolution Med Dent Sci 5(71): 2278-4748. 
7. Shaw E, Ramanakumar AV, El-Zein M, Silva FR, Galan L, et al (2016) Reproductive and genital health and risk of cervical human papillomavirus infection: results from the Ludwig-McGill cohort study 16: 116.

8. Mohamed NG, Abidin NZ, Law KS, Abe M, Suzuki M, et al. (2014) The effect of wearing sanitary napkins of different thicknesses on physiological and psychological responses in Muslim females. J Physio Anthropol 33(1): 28.

9. Alkire S (2007) The missing dimensions of poverty data: Introduction to the special issue. Oxford development studies. 35(4): 347-359.

10. Vostral SL (2011) Rely and Toxic Shock Syndrome: a technological health crisis. The Yale journal of biology and medicine 84(4): 447-459.

11. Winer RL, Hughes JP, Feng Q O’Reilly S, Kiviat NB, et al. (2006) Condom Use and the Risk of Genital Human Papillomavirus Infection in Young Women. N Engl J Med 354(25): 2645-2654.

12. Manhart, LISA E, Koutsky, Laura A (2002) Do Condoms Prevent Genital
HPV Infection, External Genital Warts, or Cervical Neoplasia? A MetaAnalysis. Sex Transm Dis 29(11): 725-735

13. Winer RL, Lee SK, Hughes JP, Adam DE, Kiviat NB, et al. (2003) Genital human papillomavirus infection: rates and risk factors in a cohort of female university students. Am J Epidemiol157(3): 218-226.

14. Grabowski MK, Gray RH, Makumbi F, Kagaayi J, Redd AD, et al. (2015) Use of injectable hormonal contraception and women's risk of herpes simplex virus type 2 acquisition: a prospective study of couples in Rakai, Uganda. Lancet Glob Health 3(8): e478-e486.

15. HoleA, Sahu A, Shaikh R, Tyagi G, Krishna CM (2017) Raman spectroscopic studies on exfoliated cells of oral and cervix. Nanophotonics Australasia 104562S.

16. Young TK, McNicol P, Beauvais J (1997) Factors associated with human papillomavirus infection detected by polymerase chain reaction among urban Canadian Aboriginal and non-Aboriginal women. Sex Transm Dis 24(5): 293-298.

Your subsequent submission with Crimson Publishers will attain the below benefits

- High-level peer review and editorial services

- Freely accessible online immediately upon publication

- Authors retain the copyright to their work

- Licensing it under a Creative Commons license

- Visibility through different online platforms

- Global attainment for your research

- Article availability in different formats (Pdf, E-pub, Full Text)

- Endless customer service

- Reasonable Membership services

- Reprints availability upon request

- One step article tracking system 